\title{
A Vector Matrices Realization of Hurwitz Algebras
}

\section{Daniel Sepunaru}

RCQCE-Research Center for Quantum Communication, Holon Academic Institute of Technology, Holon, Israel

Email: danielsepunaru@walla.co.il

How to cite this paper: Sepunaru, D. (2018) A Vector Matrices Realization of Hurwitz Algebras. Journal of Modern Physics, 9, 2370-2377.

https://doi.org/10.4236/jmp.2018.914151

Received: October 3, 2018

Accepted: December 2, 2018

Published: December 5, 2018

Copyright $\odot 2018$ by author and Scientific Research Publishing Inc. This work is licensed under the Creative Commons Attribution International License (CC BY 4.0).

http://creativecommons.org/licenses/by/4.0/

\section{(c) (i) Open Access}

\begin{abstract}
We present here a realization of Hurwitz algebra in terms of $2 \times 2$ vector matrices which maintain the correspondence between the geometry of vector spaces that is used in the classical physics and the algebraic foundation underlying quantum theory. The multiplication rule we use is a modification of the one originally introduced by M. Zorn. We demonstrate that our multiplication is not intrinsically non-associative; the realization of the real and complex numbers is commutative and associative, the real quaternions maintain associativity and the real octonion matrices form an alternative algebra. Extension to the calculus of the matrices (with Hurwitz algebra valued matrix elements) of the arbitrary dimensions is straightforward. We briefly discuss applications of the obtained results to extensions of standard Hilbert space formulation in quantum physics and to alternative wave mechanical formulation of the classical field theory.
\end{abstract}

\section{Keywords}

Hilbert Spaces, Hurwitz Algebras, Zorn Multiplication

\section{Introduction}

While the mathematical formalism of classical physics is based on use of real vector spaces, quantum physics is typically formulated algebraically. Hence, a structure that allows for a connection between both these descriptions is necessary. Among the possible algebras relevant to this task, Hurwitz algebra plays a special role. It contains one-, two-, four- and eight-dimensional quadratic normal division algebras that form the only possible numerical systems. With Hurwitz algebra, we can generate the sequence of mathematical frameworks suitable for the description of dispersion-free [1] classical field theories as well as quantum field theories that obey Heisenberg dispersion relations that use Hilbert 
modules, which are functional analytical structures similar to the usual Hilbert spaces.

\section{Matrix Treatment for Hurwitz Algebras}

Consider the subsequence of those structures with real scalar products, all of whose dynamic variables are mutually commuting and whose states are real-, complex-, quaternion- and octonion-valued [2]:

$$
(f, g)_{R} \equiv \operatorname{Tr}(f, g)
$$

where, for example, for a quaternion-valued $f$ and $g,(f, g)$ is quaternion-valued as well. The same structure may be alternatively generated by the four-dimensional vectors:

$$
\begin{gathered}
(f, g)=\operatorname{Tr}(f, g)-e_{1} \operatorname{Tr}\left\{(f, g) e_{1}\right\}-e_{2} \operatorname{Tr}\left\{(f, g) e_{2}\right\}-e_{3} \operatorname{Tr}\left\{(f, g) e_{3}\right\} \\
-e(f, g) e_{1}=\operatorname{Tr}(f, g)-e_{1} \operatorname{Tr}\left\{(f, g) e_{1}\right\}+e_{2} \operatorname{Tr}\left\{(f, g) e_{2}\right\}+e_{3} \operatorname{Tr}\left\{(f, g) e_{3}\right\} \\
-e_{2}(f, g) e_{2}=\operatorname{Tr}(f, g)+e_{1} \operatorname{Tr}\left\{(f, g) e_{1}\right\}-e_{2} \operatorname{Tr}\left\{(f, g) e_{2}\right\}+e_{3} \operatorname{Tr}\left\{(f, g) e_{3}\right\} \\
-e_{3}(f, g) e_{3}=\operatorname{Tr}(f, g)+e_{1} \operatorname{Tr}\left\{(f, g) e_{1}\right\}+e_{2} \operatorname{Tr}\left\{(f, g) e_{2}\right\}-e_{3} \operatorname{Tr}\left\{(f, g) e_{3}\right\}
\end{gathered}
$$

The sum of Equations (2), (3), (4) and (5) gives us:

$$
(f, g)_{R} \equiv \operatorname{Tr}(f, g)=\frac{1}{4}\left[(f, g)-e_{1}(f, g) e_{1}-e_{2}(f, g) e_{2}-e_{3}(f, g) e_{3}\right]
$$

or in matrix notation

$$
(\Psi, \Phi)_{R}=\frac{1}{4}\left[\bar{f},-e_{1} \bar{f},-e_{2} \bar{f},-e_{3} \bar{f}\right] *\left[\begin{array}{c}
g \\
g e_{1} \\
g e_{2} \\
g e_{3}
\end{array}\right]
$$

Similarly, the Hilbert module with a complex scalar product is generated by the sum of Equation (2) and Equation (3):

$$
(f, g)_{C} \equiv \operatorname{Tr}(f, g)-e_{1} \operatorname{Tr}\left\{(f, g) e_{1}\right\}=(f, g)-e_{1}(f, g) e_{1}
$$

In matrix notation

$$
(\Psi, \Phi)_{C}=\frac{1}{2}\left[\bar{f},-e_{1} \bar{f}\right] *\left[\begin{array}{c}
g \\
g e_{1}
\end{array}\right]
$$

The Hilbert module with complex scalar products and octonion-valued states is generated in exactly the same manner. The usual Hilbert space obviously fits that procedure. This provides evidence of the existence of a uniform matrix treatment for all Hurwitz algebras.

First of all, let us consider $2 \times 2$ matrices. We have no difficulty in representing reals, complex and real quaternions, but the underlying Cayley-Dickson procedure prevents extending the $2 \times 2$ matrix to the 8 -dimensional algebra of real octonions. In addition, the matrix obtained via Cayley-Dickson realization of real quaternions 


$$
q \Rightarrow\left(\begin{array}{cc}
q_{0}-i q_{3} & -i q_{1}-q_{2} \\
-i q_{1}+q_{2} & q_{0}+i q_{3}
\end{array}\right)
$$

yields a physically erroneous mapping of space-time geometry

$$
(x, y, z, t) \Rightarrow\left(\begin{array}{ll}
c t+z & x-i y \\
x+i y & c t-z
\end{array}\right)
$$

since it violates the assumed isotropy of the space continuum. We, therefore modify the geometric vector matrix approach originally introduced by M. Zorn [3] [4] as follows:

1) For real numbers

$$
X=x_{0} \Rightarrow\left(\begin{array}{cc}
x_{0} & 0 \\
0 & x_{0}
\end{array}\right)
$$

2) For complex numbers

$$
X=x_{0}+x_{1} i \equiv x_{0}+\vec{x} \Rightarrow\left(\begin{array}{cc}
x_{0} & \vec{x} \\
\vec{x} & x_{0}
\end{array}\right)=\left(\begin{array}{cc}
x_{0} & x_{1} i \\
x_{1} i & x_{0}
\end{array}\right)
$$

3) For quaternions

$$
X=x_{0}+\sum_{i=1}^{3} x_{i} e_{i} \equiv x_{0}+\vec{x} \Rightarrow\left(\begin{array}{cc}
x_{0} & \vec{x} \\
\vec{x} & x_{0}
\end{array}\right)=\left(\begin{array}{cc}
x_{0} & \sum_{i=1}^{3} x_{i} e_{i} \\
\sum_{i=1}^{3} x_{i} e_{i} & x_{0}
\end{array}\right)
$$

4) For octonions

$$
X=x_{0}+\sum_{i=1}^{7} x_{i} e_{i} \equiv x_{0}+\vec{x} \Rightarrow\left(\begin{array}{cc}
x_{0} & \vec{x} \\
\vec{x} & x_{0}
\end{array}\right)=\left(\begin{array}{cc}
x_{0} & \sum_{i=1}^{7} x_{i} e_{i} \\
\sum_{i=1}^{7} x_{i} e_{i} & x_{0}
\end{array}\right)
$$

and the multiplication rule is defined by

$$
\begin{aligned}
Z & =X \diamond Y \equiv\left(\begin{array}{cc}
x_{0} & \vec{x} \\
\vec{x} & x_{0}
\end{array}\right) \diamond\left(\begin{array}{cc}
y_{0} & \vec{y} \\
\vec{y} & y_{0}
\end{array}\right) \\
& =\left(\begin{array}{cc}
x_{0} y_{0}+\vec{x} \cdot \vec{y} & x_{0} \vec{y}+y_{0} \vec{x}+\vec{x} \times \vec{y} \\
x_{0} \vec{y}+y_{0} \vec{x}+\vec{x} \times \vec{y} & x_{0} y_{0}+\vec{x} \cdot \vec{y}
\end{array}\right)
\end{aligned}
$$

where

$$
\begin{gathered}
e_{i} \cdot e_{j}=-\delta_{i j} \\
\vec{x} \cdot \vec{y}=-x_{i} y_{i}=\vec{y} \cdot \vec{x} \\
\vec{x} \times \vec{y}=\varepsilon_{i j k} x_{i} y_{j} e_{k}=-\vec{y} \times \vec{x} ;
\end{gathered}
$$

$\varepsilon_{i j k}$ are structural constants in the corresponding multiplication table (see Appendix). For quaternions this is usual a totally antisymmetric three-dimensional tensor; in the case of octonions it may also be considered as a Levi-Civita tensor in seven-dimensional space.

Explicitly, for quaternions we have

$$
\vec{x} \times \vec{y}=\left(x_{2} y_{3}-x_{3} y_{2}\right) e_{1}+\left(x_{3} y_{1}-x_{1} y_{3}\right) e_{2}+\left(x_{1} y_{2}-x_{2} y_{1}\right) e_{3}
$$


and for octonions

$$
\begin{aligned}
\vec{x} \times \vec{y}= & \left(x_{2} y_{3}-x_{3} y_{2}\right) e_{1}+\left(x_{3} y_{1}-x_{1} y_{3}\right) e_{2}+\left(x_{1} y_{2}-x_{2} y_{1}\right) e_{3} \\
& +\left(x_{6} y_{5}-x_{5} y_{6}\right) e_{1}+\left(x_{6} y_{2}-x_{2} y_{6}\right) e_{4}+\left(x_{2} y_{5}-x_{5} y_{2}\right) e_{7} \\
& +\left(x_{4} y_{7}-x_{7} y_{4}\right) e_{1}+\left(x_{7} y_{2}-x_{2} y_{7}\right) e_{5}+\left(x_{2} y_{4}-x_{4} y_{2}\right) e_{6} \\
& +\left(x_{4} y_{6}-x_{6} y_{4}\right) e_{2}+\left(x_{1} y_{4}-x_{4} y_{1}\right) e_{7}+\left(x_{1} y_{6}-x_{6} y_{1}\right) e_{5} \\
& +\left(x_{5} y_{7}-x_{7} y_{5}\right) e_{2}+\left(x_{5} y_{1}-x_{1} y_{5}\right) e_{6}+\left(x_{7} y_{1}-x_{1} y_{7}\right) e_{4} \\
& +\left(x_{6} y_{7}-x_{7} y_{6}\right) e_{3}+\left(x_{7} y_{3}-x_{3} y_{7}\right) e_{6}+\left(x_{3} y_{6}-x_{6} y_{3}\right) e_{7} \\
& +\left(x_{5} y_{4}-x_{4} y_{5}\right) e_{3}+\left(x_{3} y_{5}-x_{5} y_{3}\right) e_{4}+\left(x_{4} y_{3}-x_{3} y_{4}\right) e_{5}
\end{aligned}
$$

Obviously,

$$
\vec{x} \times \vec{x}=0 \text { and } \vec{x} \cdot \vec{x}=-\sum_{i} x_{i}^{2}
$$

An involution is defined by

$$
\bar{X} \equiv x_{0}-\vec{x} \Rightarrow\left(\begin{array}{cc}
x_{0} & -\vec{x} \\
-\vec{x} & x_{0}
\end{array}\right)
$$

and this satisfies the standard requirement

$$
\overline{\bar{X}}=X
$$

(which follows immediately from (21)).

$$
\overline{X \diamond Y}=\bar{Y} \diamond \bar{X}
$$

Proof:

$$
\begin{aligned}
\overline{X \diamond Y} & =\left(\begin{array}{cc}
x_{0} y_{0}+\vec{x} \cdot \vec{y} & -x_{0} \vec{y}-y_{0} \vec{x}-\vec{x} \times \vec{y} \\
-x_{0} \vec{y}-y_{0} \vec{x}-\vec{x} \times \vec{y} & x_{0} y_{0}+\vec{x} \cdot \vec{y}
\end{array}\right) \\
\bar{Y} \diamond \bar{X} & =\left(\begin{array}{cc}
y_{0} & -\vec{y} \\
-\vec{y} & y_{0}
\end{array}\right) \diamond\left(\begin{array}{cc}
x_{0} & -\vec{x} \\
-\vec{x} & x_{0}
\end{array}\right) \\
& =\left(\begin{array}{cc}
x_{0} y_{0}+\vec{x} \cdot \vec{y} & -x_{0} \vec{y}-y_{0} \vec{x}-\vec{x} \times \vec{y} \\
-x_{0} \vec{y}-y_{0} \vec{x}-\vec{x} \times \vec{y} & x_{0} y_{0}+\vec{x} \cdot \vec{y}
\end{array}\right)
\end{aligned}
$$

Now we are in the position to prove the following statement: The algebras defined by Equations (12), (13), (14), (15), (16) and (17) are quadratic normal division algebras.

Proof:

1) $\operatorname{Tr}(X) \equiv X+\bar{X}=2 x_{0}$

2) $\operatorname{Det}(X) \equiv N(X) \equiv X \diamond \bar{X}=\left(\begin{array}{cc}x_{0} & \vec{x} \\ \vec{x} & x_{0}\end{array}\right) \diamond\left(\begin{array}{cc}x_{0} & -\vec{x} \\ -\vec{x} & x_{0}\end{array}\right)=\left(\begin{array}{cc}x_{0}^{2}-\vec{x} \cdot \vec{x} & 0 \\ 0 & x_{0}^{2}-\vec{x} \cdot \vec{x}\end{array}\right)$

Then

$$
\begin{aligned}
& X^{2}-\operatorname{Tr}(X) X+N(X) \\
& =\left(\begin{array}{cc}
x_{0} & \vec{x} \\
\vec{x} & x_{0}
\end{array}\right) \diamond\left(\begin{array}{cc}
x_{0} & \vec{x} \\
\vec{x} & x_{0}
\end{array}\right)-2 x_{0}\left(\begin{array}{cc}
x_{0} & \vec{x} \\
\vec{x} & x_{0}
\end{array}\right)+\left(x_{0}^{2}-\vec{x} \cdot \vec{x}\right) I=0
\end{aligned}
$$

From the uniqueness of the Hurwitz algebras it follows that the realization discussed above has the following properties: 
1) In one-dimensional algebra of reals and in two-dimensional algebra of complex numbers

$$
\begin{gathered}
X \diamond Y=Y \diamond X \quad \text { (commutative) } \\
X \diamond(Y \diamond Z)=(X \diamond Y) \diamond Z \quad \text { (associative) }
\end{gathered}
$$

2) In four-dimensional algebra of real quaternions

$$
X \diamond(Y \diamond Z)=(X \diamond Y) \diamond Z \quad \text { (associative) }
$$

3) In eight-dimensional algebra of real octonions

$$
\begin{aligned}
& X^{2} \diamond Y=X \diamond(X \diamond Y) \quad \text { (left alternative) } \\
& X \diamond Y^{2}=(X \diamond Y) \diamond Y \quad \text { (right alternative) }
\end{aligned}
$$

Indeed, the validity the above statements may be demonstrated through direct matrix calculations. However, as they are rather cumbersome, we will only provide the useful relations for it:

1) All Hurwitz algebras hold

$$
\begin{gathered}
\vec{x} \cdot \vec{y}=\vec{y} \cdot \vec{x} \\
\vec{x} \times \vec{y}=-\vec{y} \times \vec{x} \\
\vec{x} \cdot(\vec{y} \times \vec{z})=\vec{z} \cdot(\vec{x} \times \vec{y})=\vec{y} \cdot(\vec{z} \times \vec{x})
\end{gathered}
$$

2) For quaternions

$$
\vec{x} \times(\vec{y} \times \vec{z})=(\vec{x} \cdot \vec{y}) \vec{z}-(\vec{x} \cdot \vec{z}) \vec{y}
$$

Using relations (33) and (34) we have

$$
\begin{aligned}
& (x \diamond y) \diamond z-x \diamond(y \diamond z) \\
& =[(\vec{x} \times \vec{y}) \cdot \vec{z}-(\vec{y} \times \vec{z}) \cdot \vec{x}]+[(\vec{x} \cdot \vec{y}) \vec{z}-(\vec{y} \cdot \vec{z}) \vec{x}+(\vec{x} \times \vec{y}) \times \vec{z}-\vec{x} \times(\vec{y} \times \vec{z})] \\
& =0
\end{aligned}
$$

3) For octonions

$$
\vec{x} \times(\vec{x} \times \vec{y})=-(\vec{x} \cdot \vec{y}) \vec{x}+(\vec{x} \cdot \vec{x}) \vec{y}
$$

Using (33) for the scalar component of the alternator we have

$$
(\vec{x} \times \vec{y}) \cdot \vec{z}-(\vec{y} \times \vec{z}) \cdot \vec{x}=0
$$

Therefore,

$$
\operatorname{Tr}[(x \diamond y) \diamond z]=\operatorname{Tr}[x \diamond(y \diamond z)]
$$

Thus, calculation of scalar products in the real Hilbert module with octonion-valued states may be performed neglecting their non-associativity. Obviously, we also have

$$
\operatorname{Tr}[(x \diamond y) \diamond z]=\operatorname{Tr}[z \diamond(x \diamond y)]
$$

We have obtained the properties of associativity and commutativity which are both needed to formulate a dispersion-free field theory [2].

A detailed discussion of self-adjoint operators (dynamic variables) in those frameworks will be presented in a separate publication. 
Using (36) for the vector component of the alternator we have

$$
\begin{aligned}
& (\vec{x} \cdot \vec{x}) \vec{y}-(\vec{x} \cdot \vec{y}) \vec{x}-\vec{x} \times(\vec{x} \times \vec{y}) \\
& =(\vec{x} \cdot \vec{x}) \vec{y}-(\vec{x} \cdot \vec{y}) \vec{x}+(\vec{x} \cdot \vec{y}) \vec{x}-(\vec{x} \cdot \vec{x}) \vec{y}=0
\end{aligned}
$$

or

$$
x^{2} \diamond y=x \diamond(x \diamond y) \quad \text { (left alternative) }
$$

Similarly,

$$
\begin{aligned}
& (\vec{x} \cdot \vec{y}) \vec{y}-(\vec{y} \cdot \vec{y}) \vec{x}+(\vec{x} \times \vec{y}) \times \vec{y} \\
& =(\vec{x} \cdot \vec{y}) \vec{y}-(\vec{y} \cdot \vec{y}) \vec{x}-(\vec{x} \cdot \vec{y}) \vec{y}+(\vec{y} \cdot \vec{y}) \vec{x}=0
\end{aligned}
$$

or

$$
y \diamond x^{2}=(y \diamond x) \diamond x \quad \text { (right alternative) }
$$

Then the flexibility and the Moufang identities follow

$$
\begin{gathered}
(x \diamond y) \diamond x=x \diamond(y \diamond x) \\
(x \diamond a \diamond x) \diamond y=x \diamond[a \diamond(x \diamond y)] \\
y \diamond(x \diamond a \diamond x)=[(y \diamond x) \diamond a] \diamond x \\
(x \diamond y) \diamond(a \diamond x)=x \diamond(y \diamond a) \diamond x
\end{gathered}
$$

Consider now matrices of arbitrary dimension with matrix elements belonging to one of the Hurwitz algebras. Then the product matrix is defined by the usual multiplication rule:

$$
\begin{gathered}
\left(\begin{array}{cccc}
Z_{11} & Z_{12} & \cdots & Z_{1 n} \\
Z_{21} & Z_{22} & \cdots & Z_{2 n} \\
\vdots & \vdots & \ddots & \vdots \\
Z_{n 1} & Z_{n 2} & \cdots & Z_{n n}
\end{array}\right) \equiv\left(\begin{array}{cccc}
X_{11} & X_{12} & \cdots & X_{1 n} \\
X_{21} & X_{22} & \cdots & X_{2 n} \\
\vdots & \vdots & \ddots & \vdots \\
X_{n 1} & X_{n 2} & \cdots & X_{n n}
\end{array}\right) *\left(\begin{array}{cccc}
Y_{11} & Y_{12} & \cdots & Y_{1 n} \\
Y_{21} & Y_{22} & \cdots & Y_{2 n} \\
\vdots & \vdots & \ddots & \vdots \\
Y_{n 1} & Y_{n 2} & \cdots & Y_{n n}
\end{array}\right) \\
Z_{i j} \equiv \sum_{k=1}^{n} X_{i k} Y_{k j} ; i, j=1,2, \cdots, n
\end{gathered}
$$

where

$$
\begin{aligned}
Z_{i j} & \equiv\left(\begin{array}{cc}
Z_{i j}^{0} & \vec{Z}_{i j} \\
\vec{Z}_{i j} & Z_{i j}^{0}
\end{array}\right)=\sum_{k=1}^{n}\left(\begin{array}{cc}
x_{i k}^{0} & \vec{x}_{i k} \\
\vec{x}_{i k} & x_{i k}^{0}
\end{array}\right) \diamond\left(\begin{array}{cc}
y_{k j}^{0} & \vec{y}_{k j} \\
\vec{y}_{k j} & y_{k j}^{0}
\end{array}\right) \\
& \equiv \sum_{k=1}^{n}\left(\begin{array}{cc}
x_{i k}^{0} y_{k j}^{0}+\vec{x}_{i k} \cdot \vec{y}_{k j} & x_{i k}^{0} \vec{y}_{k j}+y_{k j}^{0} \vec{x}_{i k}+\vec{x}_{i k} \times \vec{y}_{k j} \\
x_{i k}^{0} \vec{y}_{k j}+y_{k j}^{0} \vec{x}_{i k}+\vec{x}_{i k} \times \vec{y}_{k j} & x_{i k}^{0} y_{k j}^{0}+\vec{x}_{i k} \cdot \vec{y}_{k j}
\end{array}\right)
\end{aligned}
$$

$\forall X_{i k}, Y_{i k}, Z_{i k}$ elements of $\mathrm{R}, \mathrm{C}, \mathrm{H}$ and $\mathrm{O}$ algebras.

Thus, the product matrix is defined as the usual sum of pairs of multipliers and the product of each pair is defined by the vector multiplication introduced above. The trace and determinant of the product matrix are always real and are defined according to the usual rules. For example,

$$
\operatorname{Tr}(Z)=\sum_{i=1}^{n} Z_{i i}^{0}
$$

Therefore, the result of the calculation is unambiguous. 


\section{Conclusions}

Having discussed the geometric extension of conventional matrix multiplication which is uniformly valid for all quadratic normal division algebras, I would like, in conclusion, to emphasize that the suggested matrix realization is of crucial importance for quaternion and octonion extensions of standard functional analysis since the real as well as the complex Hilbert modules require the use of multicomponent states. The results obtained allow for the introduction and investigation of the operators necessary for the description of the system dynamics as well as for the observables (self-adjoint operators) [5] [6]. In addition, the transition from the vector matrix to the standard one may provide an alternative mechanism for spontaneous breakdown of internal symmetries as suggested by the comparison of Equations (10) and (14). Historically, the multiplication operation over real numbers was first extended to physically relevant three-dimensional space and only later to spaces of arbitrary dimensions and signatures [7]. The invention of scalar matrix multiplication was an alternative to this generalization. It seems reasonable to expect that the vector matrix multiplication suggested here may be extended to additional types of algebras (Clifford, Lie, Jordan, etc.), but that lies outside the scope of this investigation.

\section{Conflicts of Interest}

The author declares no conflicts of interest regarding the publication of this paper.

\section{References}

[1] von Neumann, J. (1931) Mathematische Grundlagen der Quantenmechanik. Springer, Berlin.

[2] Sepunaru, D. (2006) quant-ph/0606121.

[3] Zorn, M. (1930) Abhandlungen aus dem Mathematischen Seminar der Universität Hamburg, 8, 123-147.

[4] Zorn, M. (1933) Abhandlungen aus dem Mathematischen Seminar der Universität Hamburg, 9, 395-402.

[5] Dirac, P.A.M. (1958) The Principles of Quantum Mechanics. 4th Edition, Clarendon Press, Oxford.

[6] Doughty, N.A. (1990) Lagrangian Interaction. Addison-Wesley, Boston.

[7] Cartan, E. (1938) Lecons sur la theorie des spineuers. Actualitesscientifiques et industrielles, Hermann, Paris, 643, 701. 


\section{Appendix}

For readers who would like to verify the statements in this paper by direct calculation, I reproduce here the multiplication tables of Hurwitz algebra.

Table A1. Complex numbers.

\begin{tabular}{ccc}
\hline & $e_{0}$ & $e_{1}$ \\
\hline$e_{0}$ & $e_{0}$ & $e_{1}$ \\
$e_{1}$ & $e_{1}$ & $-e_{0}$ \\
\hline
\end{tabular}

Table A2. Quaternions.

\begin{tabular}{ccccc}
\hline & $e_{0}$ & $e_{1}$ & $e_{2}$ & $e_{3}$ \\
\hline$e_{0}$ & $e_{0}$ & $e_{1}$ & $e_{2}$ & $e_{3}$ \\
$e_{1}$ & $e_{1}$ & $-e_{0}$ & $e_{3}$ & $-e_{2}$ \\
$e_{2}$ & $e_{2}$ & $-e_{3}$ & $-e_{0}$ & $e_{1}$ \\
$e_{3}$ & $e_{3}$ & $e_{2}$ & $-e_{1}$ & $-e_{0}$ \\
\hline
\end{tabular}

Table A3. Octonions.

\begin{tabular}{ccccccccc}
\hline & $e_{0}$ & $e_{1}$ & $e_{2}$ & $e_{3}$ & $e_{4}$ & $e_{5}$ & $e_{6}$ & $e_{7}$ \\
\hline$e_{0}$ & $e_{0}$ & $e_{1}$ & $e_{2}$ & $e_{3}$ & $e_{4}$ & $e_{5}$ & $e_{6}$ & $e_{7}$ \\
$e_{1}$ & $e_{1}$ & $-e_{0}$ & $e_{3}$ & $-e_{2}$ & $e_{7}$ & $-e_{6}$ & $e_{5}$ & $-e_{4}$ \\
$e_{2}$ & $e_{2}$ & $-e_{3}$ & $-e_{0}$ & $e_{1}$ & $e_{6}$ & $e_{7}$ & $-e_{4}$ & $-e_{5}$ \\
$e_{3}$ & $e_{3}$ & $e_{2}$ & $-e_{1}$ & $-e_{0}$ & $-e_{5}$ & $e_{4}$ & $e_{7}$ & $-e_{6}$ \\
$e_{4}$ & $e_{4}$ & $-e_{7}$ & $-e_{6}$ & $e_{5}$ & $-e_{0}$ & $-e_{3}$ & $e_{2}$ & $e_{1}$ \\
$e_{5}$ & $e_{5}$ & $e_{6}$ & $-e_{7}$ & $-e_{4}$ & $e_{3}$ & $-e_{0}$ & $-e_{1}$ & $e_{2}$ \\
$e_{6}$ & $e_{6}$ & $-e_{5}$ & $e_{4}$ & $-e_{7}$ & $-e_{2}$ & $e_{1}$ & $-e_{0}$ & $e_{3}$ \\
$e_{7}$ & $e_{7}$ & $e_{4}$ & $e_{5}$ & $e_{6}$ & $-e_{1}$ & $-e_{2}$ & $-e_{3}$ & $-e_{0}$ \\
\hline & & & & & & & &
\end{tabular}

